\title{
A Multi-objective Optimization Algorithm for Multiple Home Users Intelligent Power Management and Control Based on Pareto and Nash Equilibrium Game
}

\author{
Zhou $\mathrm{Yu}^{1}, \mathrm{Hu}$ Weifeng ${ }^{1}$, Wang Dezhi ${ }^{2,}{ }^{*}, X u \mathrm{Zheng}^{1}, \mathrm{Yu} \mathrm{Tao}^{2}$ \\ ${ }^{1}$ Yancheng Power Supply Company, State Grid Jiangsu Electric Power Company, 224005 Yancheng, China \\ ${ }^{2}$ School of Electric Power South China University of Technology, 510640 Guangzhou, China
}

\begin{abstract}
A multi-objective optimization model for multiple home users intelligent power management and control is proposed. A photovoltaic power model, an electric vehicle battery model and a load model are developed first, and then a strategy of home intelligent power management is presented based on battery operation and PV spontaneous self-use. Secondly, a multi-objective optimization model of multiple home users intelligent power management, including the user comfort, economy and optimization of load curve, is provided under the constraints. Then using a multi-objective optimization algorithm and Nash equilibrium game theory to solve the multi-objective problem. Finally, the 100-home power management and control simulation case show that the presented algorithm can improve the comfort and the economy of users effectively, but also help the power grid to peak load shifting.
\end{abstract}

\section{Introduction}

In recent years, with the popularization and development of distributed generation supply and electric vehicle, many scholars have launched research on all aspects of the active distribution network [1,2]. Some enterprises and institutions also have begun to undertake demonstration projects [3]. The technology of active distribution network through scientific measures to guide users to use electricity effectively, and to optimize the allocation of resources, achieving the minimum cost of the intelligent use of electricity [4]. How to realize efficient management and control of multiple home users based on active distribution network is a question worthy of discussion.

In developing power plans, [5] taking the diversity of user requirements and multiple characteristics of home users into account, set the optimal scheduling model for different optimization targets. [6] was proposed a method of converting the user comfort to electricity cost, and introduced an effective management strategy for battery charging and discharging. [7] gives out a detailed processing method of the room temperature, the water temperature regulation and different types of electric equipment. In dealing with objective function, [8] converting the user's electricity consumption behaviour to electricity cost, and considering the optimization target of users' electric charge and electricity satisfaction.

Compared with the optimization of single home user, the optimization of multiple home users has some characteristics including large total load, appliances diversification, electricity consumption habits and so on. At the same time, the rapid development of active distribution network, more distributed generation and electric vehicle access to home users, which increase the complexity of home energy management system (HEMS). Therefore, this paper builds a multi-objective optimization model for multiple home user management and control based on active distribution network. Firstly, the home users are classified according to similar of the electrical equipment. Then, the neighbour immune algorithm non-dominated multi-objective optimization algorithm (NNIA) based on Pareto Nash equilibrium is proposed to solve the problem.

\section{Classification and system model}

\subsection{Classification of multiple home users}

In this paper, the multiple home users are divided into different types, and the criteria of the classification are mainly considering the different types of photovoltaic, electric vehicles and household controllable power equipment. Multiple home users can be divided into the following three categories:

1) I-type: Both PV and energy storage.

2) II-type: Energy storage only.

3) III-type: Without PV and energy storage. 
In single home, equipment divided into two parts, controllable system and uncontrollable system. The controllable system includes storage systems, room temperature regulating system, water temperature control systems, and PV system. The uncontrollable system mainly includes basic household load, such as lighting, refrigerator, kitchen appliances, and so on.

\subsection{System model}

The proportion of households with different types will affect the optimization results, especially reducing the proportion of energy storage systems will reduce the load on the peak load shifting effect. PV and energy storage equipment will affect the household electricity consumption, electricity. The PV system, by cooperating with energy storage system, users can store the photovoltaic energy, realizing spontaneous self-use and the maximum economic benefits. The controllable load through reasonable scheduling to meet the needs of users' living comfort.

1) Energy storage system model: Energy storage of electric vehicles is mainly using lithium battery which keeps constant power of charging and discharging [9].

$$
Q_{\mathrm{B}}(h)=Q_{\mathrm{B}}(h-1)+\delta_{\mathrm{ch}} * P_{\mathrm{ch}}(h)-1 / \delta_{\mathrm{dch}} * P_{\mathrm{dch}}(h)
$$

where $Q_{\mathrm{B}}(h)$ is the remaining capacity of battery; $P_{\mathrm{ch}}(h)$ and $P_{\mathrm{dch}}(h)$ are the charge power and discharge power of battery; $\delta_{\text {ch }}$ and $\delta_{\text {dch }}$ are charge-discharge efficiency of battery.

2) PV system model: The PV output power is related to solar irradiance and air temperature. We can obtain output prediction curve of each home users the next day by the day-ahead prediction and historical data of solar irradiance and air temperature.

3) Room temperature regulating system (RTRS) model: The mathematical model of room temperature regulation is established as follows $[10,11]$.

$$
\begin{aligned}
& T_{\mathrm{H}}(h+1)=e^{-1 / R C} * T_{\mathrm{H}}(h)+ \\
& \quad R\left(1-e^{-1 / R C}\right) * P_{\mathrm{H}}(h)+\left(1-e^{-1 / R C}\right) * T_{\text {out }}(h)
\end{aligned}
$$

where $R$ is the room thermal resistance; $C$ is the room heat capacity; $T_{\text {out }}(h)$ is the outdoor temperature; $P_{\mathrm{H}}(h)$ is the temperature regulation power.

4) Water temperature regulation system (WHRS) model: The discrete mathematical model of water temperature regulation can be described as follows [11].

$$
\begin{aligned}
& \mathrm{c} \rho T_{\mathrm{w}}(\mathrm{h}+1)=\left(1-\alpha_{\mathrm{w}}\right) *\left\{\varphi P_{\mathrm{w}} \mathrm{u}_{\mathrm{w}}(\mathrm{h})+\right. \\
& \left.\quad\left[\mathrm{c} \rho\left[V_{0}-V_{\mathrm{u}}(h)\right] T_{\mathrm{w}}(h)+V_{\mathrm{u}}(h) T_{\mathrm{w} 0}(h)\right]\right\}
\end{aligned}
$$

where $\alpha_{\mathrm{w}}$ is the water energy loss coefficient; $\varphi$ is energy exchange coefficient; $V_{0}$ is the volume of the water tank; $V_{\mathrm{u}}(h)$ is the water consumption; $T_{\mathrm{w} 0}(h)$ is the temperature of tap water; $P_{\mathrm{W}}$ is the heating power; $u_{\mathrm{w}}(h)$ is the water heater switch state; $c$ and $\rho$ are the specific heat capacity and the density of water.

\section{Multi-objective optimization model for multiple home users intelligent power management and control}

\subsection{Optimization objective function}

1) Objective function of user side: For home users, the indoor temperature and the hot water temperature are the state variables to achieve comfort and economic optimization.

a) Economic objective: The economic target is to lowest the electric charge by adjusting the PV and battery based on TOU.

b) Comfort objective: The comfort target of home users is to minimize the deviation between the actual room temperature and the water temperature with the expected value under the constraints of home comfort.

$$
\begin{cases}\min & F=\sum_{i=1}^{N_{\mathrm{h}}}\left\{\sum _ { h = 1 } ^ { N _ { \mathrm { c } } } \left\{v(i, h) * f_{\mathrm{H}}\left(T_{\mathrm{H}}(i, h)\right)+\right.\right. \\ & \left.\left.g(i, h) * f_{\mathrm{W}}\left(T_{\mathrm{W}}(i, h)\right)\right\}\right\} \\ \text { s.t. } \quad & f_{\mathrm{H}}\left(T_{\mathrm{H}}(i, h)\right)=\left(T_{\mathrm{H}}(i, h)-S H(i, h)\right)^{2} \\ & f_{\mathrm{W}}\left(T_{\mathrm{W}}(i, h)\right)=\left(T_{\mathrm{W}}(i, h)-S W(i, h)\right)^{2} \\ & f_{\mathrm{H}}\left(T_{\mathrm{H}}(i, h)\right) \leq f_{\mathrm{H} \cdot \max } \\ & f_{\mathrm{W}}\left(T_{\mathrm{W}}(i, h)\right) \leq f_{\mathrm{W} \cdot \max }\end{cases}
$$

where $F$ is objective function of comfort; $N_{\mathrm{h}}$ is the home users number; $v(i, h)$ and $g(i, h)$ are penalty function; $S H(i, h)$ and $S W(i, h)$ are the expected value of the room temperature and the water temperature.

2) Objective function of grid side: For the grid side, the smaller the fluctuation of load curve is, the better it is. The standard deviation of the load curve is used as the optimization goal.

$$
\min \sigma=\sqrt{\frac{1}{N_{\mathrm{c}}} \sum_{h=1}^{N_{\mathrm{c}}}\left(\sum_{i=1}^{N_{\mathrm{h}}} P_{\text {grid }}(i, h)-\mu\right)^{2}}
$$

where $\sigma$ is the standard deviation of the load curve; $P_{\text {grid }}(i, h)$ is the exchange power; $\mu$ is the average load.Captions should be typed in 9-point Times. They should be centred above the tables and flush left beneath the figures.

\subsection{Constraints}

1) Constraints for power balance: Power exchange between the grid and the home users must meet the law of conservation of energy.

$P_{\text {grid }}(h)=P_{\text {Batt }}(h)+P_{\mathrm{H}}(h)+P_{\mathrm{w}} * u_{\mathrm{w}}(h)+P_{\mathrm{o}}(h)-P_{\mathrm{PV}}(h)(6)$ where $P_{\text {Batt }}(h)$ is the power of battery; $P_{\mathrm{o}}(h)$ is the basic load; $P_{\mathrm{PV}}(h)$ is the PV power.

2) Constraints for energy storage system: In addition to meet the maximum charging and discharging power, considering the battery life, the minimum use capacity shall not be less than $30 \%$.

$$
\begin{gathered}
0 \leq \frac{P_{\text {ch }}(h)}{\delta_{\text {ch }}} \leq P_{\text {ch.max }} \\
0 \leq P_{\text {dch }}(h)_{*} \delta_{\text {dch }} \leq P_{\text {dch.max }} \\
Q_{\text {B.L }} \leq Q_{\text {В }}(h) \leq Q_{\text {B.U }}
\end{gathered}
$$

where $P_{\text {ch.max }}$ and $P_{\text {dch.max }}$ are charging/discharging power limit; $Q_{\mathrm{B} . \mathrm{U}}$ and $Q_{\mathrm{B} . \mathrm{L}}$ are the limit of battery. 
3) Constraints for the room temperature and the water temperature regulating system:

$$
\begin{gathered}
0 \leq P_{\mathrm{H}}(h) \leq P_{\mathrm{H} \cdot \mathrm{U}} \\
u_{\mathrm{W}}(h) \in\{0,1\} \\
0 \leq T_{\mathrm{H}}(h) \leq T_{\mathrm{H} \cdot \mathrm{U}} \\
0 \leq T_{\mathrm{W}}(h) \leq T_{\mathrm{W} \cdot \mathrm{U}}
\end{gathered}
$$

where $P_{\mathrm{H} . \mathrm{U}}$ is the maximum power of air conditioners; $T_{\mathrm{H} . \mathrm{U}}$ and $T_{\mathrm{W} . \mathrm{U}}$ are the upper limit of room temperature and water temperature.

\section{Multi-objective optimization algorithm for multiple home users management and control}

\subsection{Pareto theory and NNIA algorithms}

The multi-objective optimization problem based on Pareto theory can be formulated as:

$$
\begin{cases}\text { Min } & F(X)=F_{i}(X) i=1,2, \ldots, M_{\text {obj }} \\ \text { s.t. } & g_{i}(X) \leq 0, \quad i=1,2, \ldots, M_{\text {ineq }} \\ & h_{i}(X)=0, \quad i=1,2, \ldots, M_{\text {eq }}\end{cases}
$$

Where $F_{i}$ is the $i$ th objective; $X$ is the decision variables; $M_{o b j}, M_{\text {ineq }}$ and $M_{e q}$ is the objective number of inequality constraints and equality constraints.

This paper using a multi-objective algorithm based on artificial immune, which called NNIA, to solve the above optimization model. NNIA saves non-dominated individuals which have been found until now in an outer population, called the dominant population. Only part of rare non-dominated individuals, are selected to do cloning, recombination, and mutation. This strengthens the search for the sparse part of the POF, so as to find the optimal Pareto.

\subsection{Nash Equilibrium-based Decision Making}

After obtaining the final POF, it is necessary to select an optimal compromise solution as the final decision. In this paper, the competing objectives can be seen like the noncooperative decision makers. Therefore, by using the multi-objective decision model based on Nash equilibrium to solve the optimal compromise solution, in order to obtain the corresponding optimal electricity plan. The multi-objective decision model based on Nash equilibrium can be described as follows:

$$
\begin{aligned}
& \left(\operatorname{Max} \operatorname{NASH}\left(H_{1}, \ldots H_{j}, \ldots H_{M_{e_{i}}}, u_{1}, \ldots u_{i}, \ldots u_{M_{w_{4}}}\right)\right. \\
& =\sum_{i=1}^{M_{\infty}}\left(\sum_{j=1}^{M_{s i}}\left(-\omega_{i} F_{i}\right)\left(\prod_{i=1}^{M_{t+1}} h_{y}\right)\right)-\sum_{i=1}^{M_{i+1}} v_{t} \\
& \text { s.t. } \sum_{j=1}^{M_{p}} h_{\theta}=1, i=1,2,3, \ldots, M_{\text {otj }} \\
& h_{0} \geq 0, \quad i=1,2,3, \ldots, M_{\text {odg }}, j=1,2,3, \ldots, M_{v f} \\
& \sum_{j=1}^{M_{t_{1}}}\left(-\omega_{i} F_{v}\right) h_{j} \leq v_{i}, \quad i=1,2,3, \ldots, M_{\alpha j}
\end{aligned}
$$

Where $H_{i}$ is the equilibrium value of the $i$ th objective; $f_{i j}$ is the $i$ th objective's normalized fitness of the $j$ th element in the POF; $v_{i}$ expresses the upper limit of the expectation of fitness values for the $i$ th objective player; $M_{\text {pof }}$ is the prespecified size to represent the limited POF set; $\omega_{i}$ is the weight for the relative importance of the $i$ th objective function.

The list of equilibrium values can be provided for the decision maker, and the optimal compromise solution can then be gained by computing the best joint equilibrium which represents the highest payoff outcome obtained from this joint action, as (16).

$$
\max \left[\prod_{i=1}^{M_{\mathrm{obj}}} h_{i 1}, \prod_{i=1}^{M_{\mathrm{obj}}} h_{i 2}, \ldots \prod_{i=1}^{M_{\mathrm{obj}}} h_{i j}, \ldots \prod_{i=1}^{M_{\mathrm{obj}}} h_{i \mathrm{M}_{\mathrm{pof}}}\right]
$$

\section{Simulation Case}

\subsection{Simulation parameter settings}

For the model of multiple home power management, the 100 households system is employed. Home users use the typical daily electricity consumption habits, and assuming that each home user can completely response the demand of power grid. In addition, the battery, photovoltaic power generation, room temperature regulation and water temperature regulation and other parameters as shown in Table 1.

Table 1. Parameter settings

\begin{tabular}{cc}
\hline Maximum output of PV & $2 \mathrm{~kW}$ \\
Battery energy capacity & $5.9 \mathrm{~kW} \cdot \mathrm{h}$ \\
Battery power limit & $3 \mathrm{~kW}$ \\
Charge and discharge & $90 \% / 90 \%$ \\
efficiency & $30 \%$ \\
Battery capacity limit & $2 \mathrm{~kW}$ \\
RTRS maximum power & $80 \mathrm{~L}$ \\
Tank capacity & $1 \mathrm{~kW}$ \\
\hline WHRS power & \\
\hline
\end{tabular}

\subsection{Pareto theory and NNIA algorithms}

This paper divides the 100 home users into different types of proportion respectively, and divides into three kinds of cases, as shown in Table 2.

Table 2. The proportion of different types of homes

\begin{tabular}{clll}
\hline $\begin{array}{c}\text { Home } \\
\text { type }\end{array}$ & I-type & II-type & III-type \\
\hline Case 1 & 50 & 25 & 25 \\
Case 2 & 25 & 50 & 25 \\
Case 3 & 25 & 25 & 50 \\
\hline
\end{tabular}

1) Nash equilibrium decision making: A set of optimal solution will obtain under every case, and the Nash equilibrium method is used to select out the best compromise solution as final decision solution. Specific reference Figure 1 is given under the Case 1.

2) Analysis on simulation results of home comfort: From the box whisker plots of different case, as shown in Figure 2 to Figure 4, we can see that the room 
temperature and the water temperature are basically near the expected value. It shows that the model has little effect on the users' comfort under different cases, and it indicates that the proposed algorithm can satisfy the users' demand for comfort.

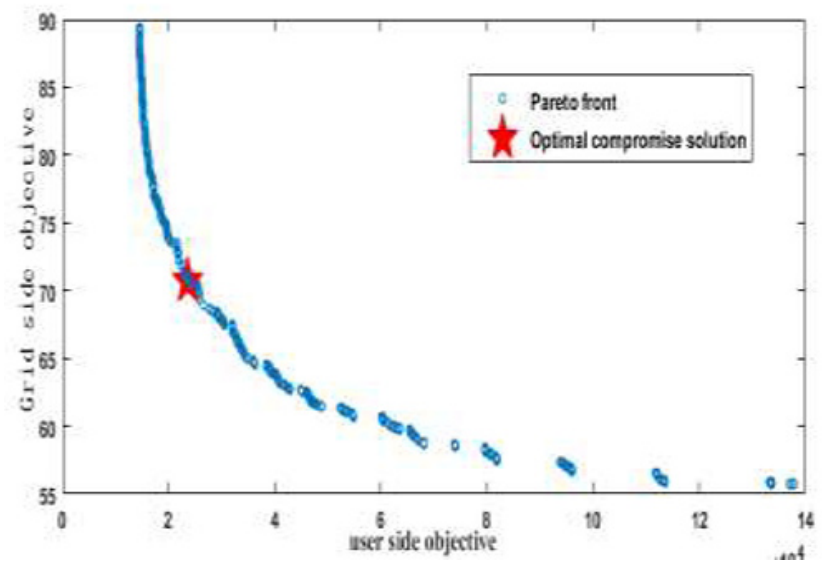

Fig 1. Pareto curves and the optimal compromise solution

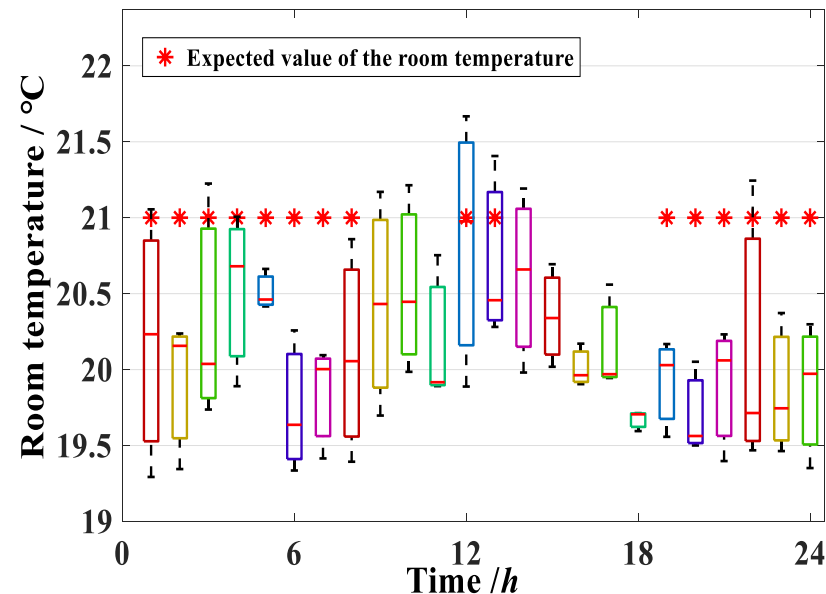

(a) Case 1 room temperature

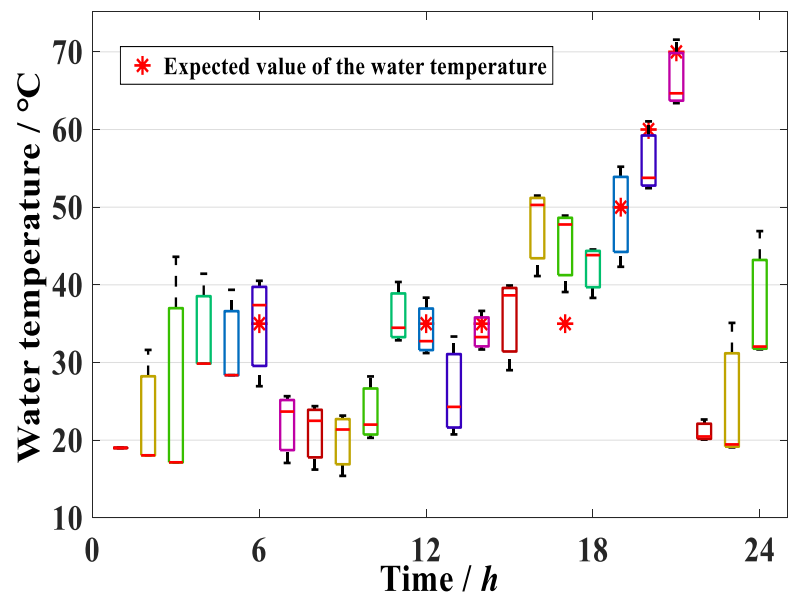

(b) Case 1 water temperature

Fig 2. The box-and-whisker plot of the water temperature and the room temperature obtained under case 1

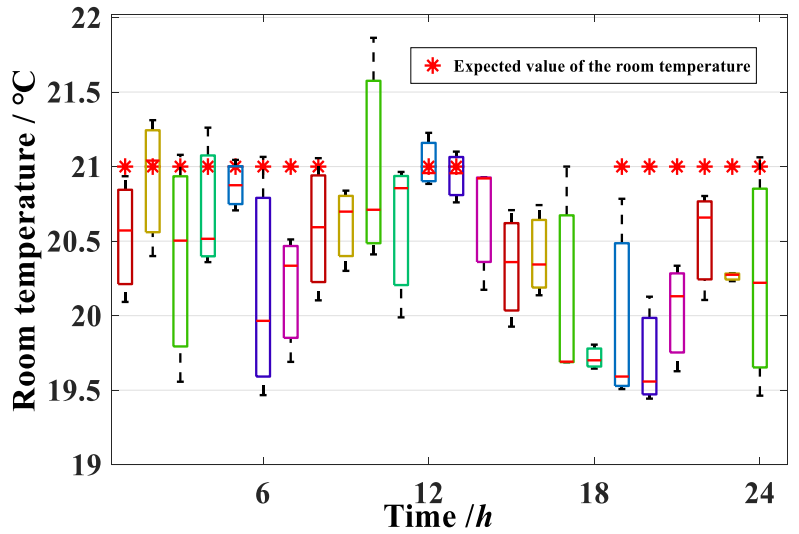

(a) Case 2 room temperature

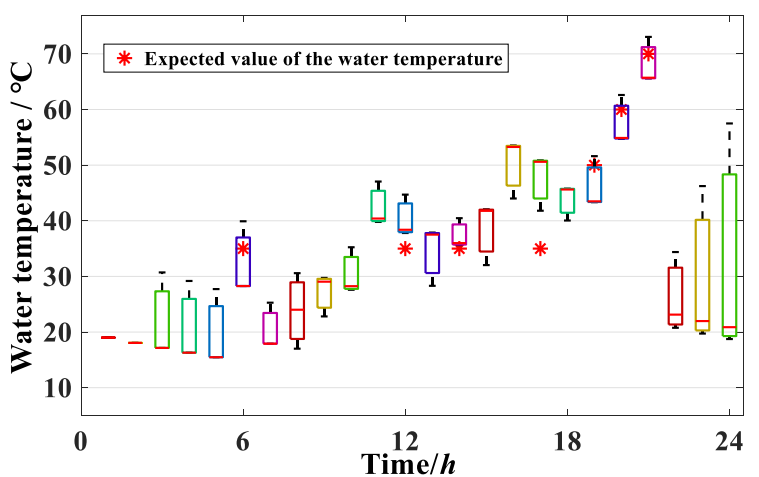

(b) Case 2 water tempeature

Fig 3. The box-and-whisker plot of the water temperature and the room temperature obtained under case 2

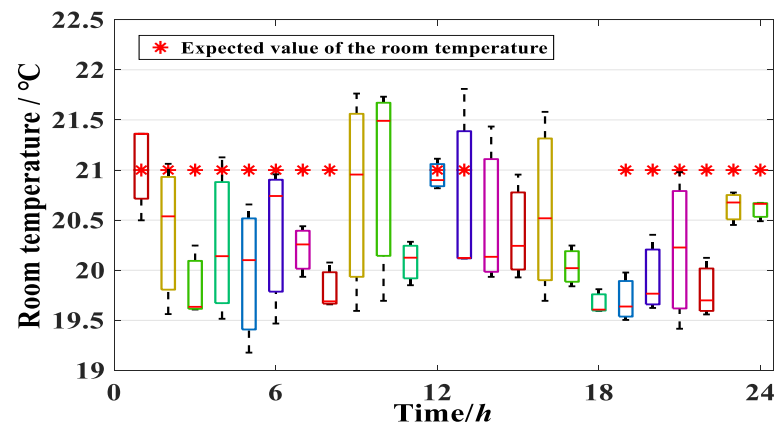

(a) Case 3 room temperature

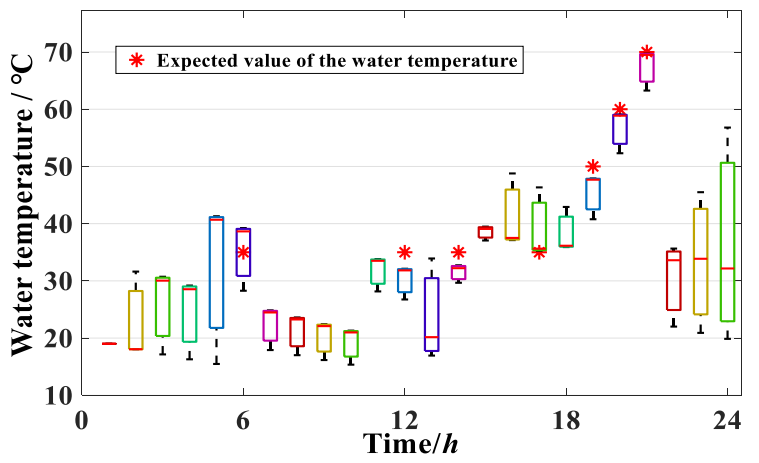

(b) Case 3 water temperature

Fig 4. The box-and-whisker plot of the water temperature and the room temperature obtained under case 3 


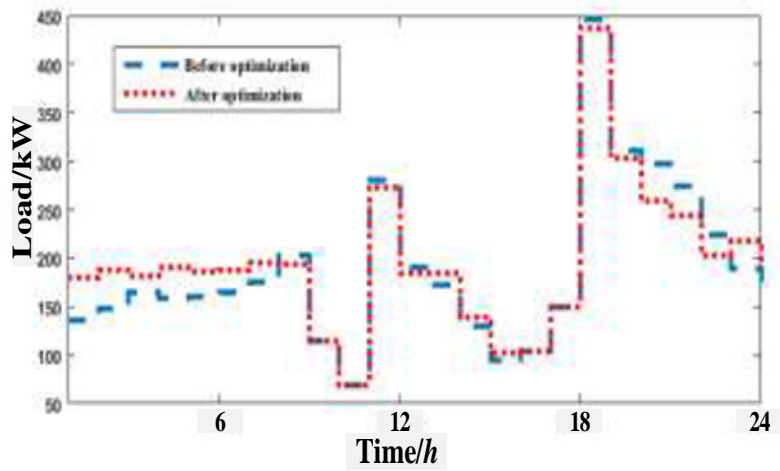

(a) Case 1

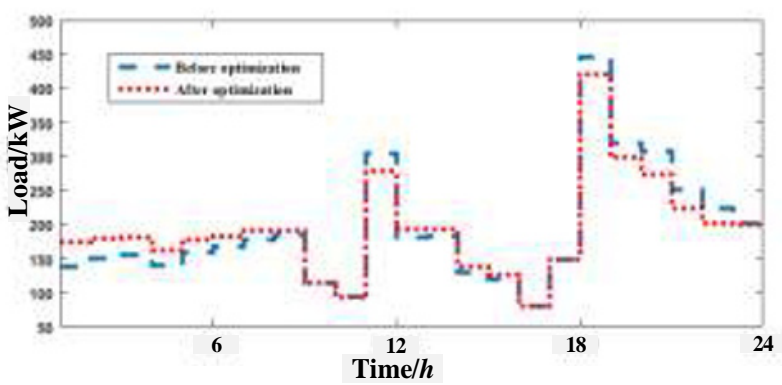

(b) Case 2

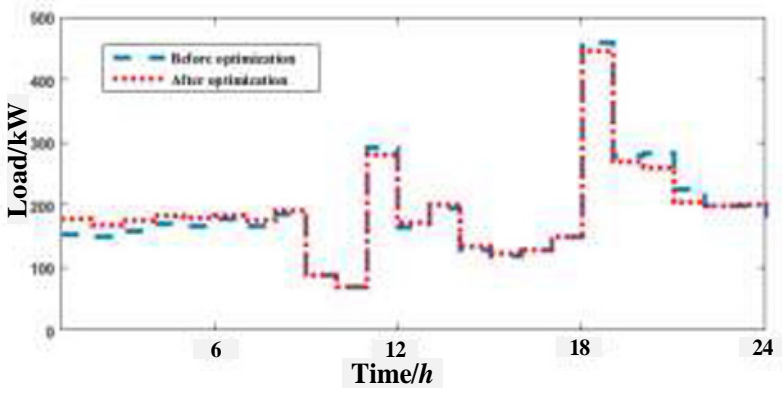

(c) Case 3
From Figure 5 we can see that the three cases play a role in suppressing the load fluctuation and peak load shifting while the Case 2 and Case 3 have more storage equipment, for the load transfer has played a greater role.

Table 3 lists the simulation results of three case. As can be seen from table horizontal data, Case 1 is significantly better than the other case in energy storage; Case 2 in the peak load shifting and restrain the load curve fluctuations has the best effect. From the longitudinal data and the simulation process, it shows that the NNIA algorithm has an excellent performance in the convergence speed and the computing model.

\section{Conclusions}

In this paper, a multi-objective optimization algorithm based on Pareto Nash equilibrium game of multiple home users power management is developed. Through the interaction between the family and the power grid, the photovoltaic power generation, electric vehicle access and family load collaborative scheduling to achieve the user electricity comfort, economy and electric network side load curve optimization. The NNIA algorithm is used to solve the Pareto front and the optimal tradeoff is obtained by using the Nash equilibrium game theory. The simulation results show that the multi-objective optimization algorithm based on multiple home users can satisfy the user comfort and economy, and can also meet the load curve optimization of power grid.

This work is support by Science and Technology Project of State Grid Corporation of China (Research on Demand Strategies of Multi-Source Interconnected Distribution Network and Diversified Power Consumption in Energy Internet, No. 5210A0180004).

Figure 5. The simulation results of different cases

3) Analysis of simulation results of load curve optimization:

Table 3 Simulation result data of all kinds of case

\begin{tabular}{|c|c|c|c|c|c|c|c|}
\hline \multirow{2}{*}{ Parameter } & \multirow{2}{*}{ Algorithm } & \multicolumn{3}{|c|}{ Before optimization } & \multicolumn{3}{|c|}{ After optimization } \\
\hline & & Case 1 & Case 2 & Case 3 & Case 1 & Case 2 & Case 3 \\
\hline \multirow{3}{*}{$Q_{\text {store }} / \mathrm{kW} \cdot \mathrm{h}$} & NNIA & & & & 84.4581 & 75.2136 & 47.9684 \\
\hline & SPEA-II & 0 & 0 & 0 & 132.9677 & 76.8844 & 75.9208 \\
\hline & NSGA- II & & & & 114.8736 & 133.1312 & 67.2126 \\
\hline \multirow{3}{*}{$\sigma / \mathrm{kW}$} & NNIA & & & & 72.6305 & 68.0754 & 73.9184 \\
\hline & SPEA-II & 86.2901 & 84.9017 & 88.7004 & 68.9126 & 64.5890 & 72.9779 \\
\hline & NSGA-II & & & & 76.1925 & 74.4978 & 81.1101 \\
\hline \multirow{3}{*}{$\Delta \mathrm{P}_{\mathrm{p}-\mathrm{p}} / \%$} & NNIA & & & & 77.54 & 76.83 & 78.16 \\
\hline & SPEA-II & 79.89 & 84.36 & 84.79 & 77.58 & 72.13 & 77.59 \\
\hline & NSGA-II & & & & 79.37 & 83.45 & 84.40 \\
\hline \multirow{3}{*}{ Savefee/¥ } & NNIA & & & & 29.01 & 36.30 & 19.31 \\
\hline & SPEA-II & 0 & 0 & 0 & 15.85 & 33.49 & 10.55 \\
\hline & NSGA-II & & & & 25.42 & 6.68 & 16.49 \\
\hline
\end{tabular}




\section{References}

1. Y. You, D. Liu, W. Yu, F. Chen, F. Pan, Automat. Electron. Power Sys. 36(18), pp10-16 (2012)

2. B. Zhao, C. Wang, J. Zhou, J. Zhao, Y. Yang, Automat. Electron. Power Sys., 38(18), pp125-135 (2014)

3. D. Liu, Y. Chen, Y. Huang, Y. You, W. Yu, Proc. Chin. Soc. Electrical Eng., 34(31), pp5500-5506 (2014)

4. W. Wang, G. He, J. Wan, W. Yang, Y. Chen, Automat. Electron. Power Sys., 36(3), pp10-15 (2012)

5. J. Liu, R. He, R. Li, G Zeng, Application Research of Computers, 32(1), pp132-137 (2015)
6. Y. Iwafune, T. Ikegami, JGDSF Jr, T. Oozeki, K. Ogimoto, Energy Convers. Manage., 96, pp322-329 (2015)

7. Y. Huang, H. Tian, L. Wang, Int. J. Elec. Power., 73, pp448-455 (2015)

8. M. A. A. Pedrasa, T. D. Spooner, I. F. Macgill, IEEE T. Smart Grid, 1(2), pp134-143 (2010)

9. X. Wang, C. Shao, X. Wang, C. Du, Proc. Chin. Soc. Electrical Eng., 33(1), pp1-10 (2013)

10. S. Bracco, F Delfino, F. Pampararo, M. Robba, M. Rossi, Energy Convers. Manage., 96, pp511-520 (2015)

11. S. Shao, M. Pipattanasomporn, S. Rahman, IEEE T. Power Electr., 28(2), pp607-614 (2013) 